\title{
CFD study of the thermal transfer of a non-Newtonian fluid within a tank mechanically stirred by an anchor-shaped impeller
}

\author{
L. Rahmani ${ }^{1}$, O. Seghier ${ }^{1}$, A. Benmoussa ${ }^{1}$ and B. Draoui ${ }^{1}$ \\ ${ }^{1}$ Arids zones energetic laboratory (ENERGARID), TAHRI Mohamed University of Béchar, BP 417, 08000 Béchar, \\ Algeria.
}

\begin{abstract}
The most of operations of chemical, biochemical or petrochemical industries are carried out in tanks or in reactors which are mechanically-controlled. The optimum mode of operation of these devices requires a finalized knowledge of the thermo-hydrodynamic behavior induced by the agitator. In the present work, the characterization of the incompressible hydrodynamic and thermal fields of a non-Newtonian fluid (Bingham) in a flat, non-baffled cylindrical vessel fitted with anchor agitator was undertaken by numerical simulation, using the CFD code Fluent (6.3.26) based on the finite volume discretization method of the energy equation and the Navier-Stokes equations which are formulated in (U.V.P) variables. We have summarized this simulated system by comparing of the consumed power and the Nusselt number for this type of mobile (Anchor agitator).
\end{abstract}

\section{Introduction}

Many industries (chemical, pharmaceutical and food) use, produce or transform complex fluids. These are high viscosity fluids, often with non-Newtonian behaviour. Their preparation generally requires a heating step followed by a cooling step. Heating is essential to facilitate manufacture (chemical reaction and / or physical-chemical transformation).

The cooling or the finishing step consists in bringing the fluids to a temperature close to ambient temperature in order to approach the conditioning step. The cooling is most often carried out in the same stirred tank.

There are two strong demands in the industry: firstly, ensure the quality of finished products. Secondly, work in a more and more concentrated environment for pollution and cost problems. In order to know and check all the involved aspects in the process, in particular hydrodynamics and heat transfer.

The objective of this work is the thermal study of incompressible laminar flows in a tank mechanically agitated by an anchor agitator and the fluids used are viscoplastic with Bingham model. The modeling difficulties of the problem lie in the viscoplastic nature of the fluid. We perform a numerical simulation of hydrodynamics and heat transfer using the industrial code (Fluent ${ }^{\circledR}$ ). The results obtained give us the possibility to make a comparative study between our results and previous work from the point of view of energy consumption (Power Number), and heat transfer (Nusselt number).

\section{Numerical models}
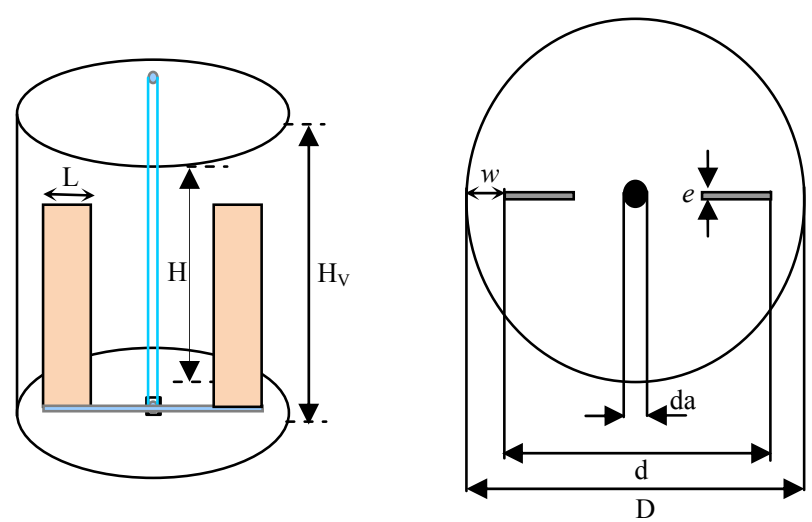

Fig. 1. The geometry of mixing system.

The mixing systems are presented in Fig1. The tank is a flat-bottomed vessel (inside diameter: D) equipped with an anchor agitator (diameter $\mathrm{d}$ ). $\mathrm{H}$ is the fluid height. Values for the agitator width ' $\mathrm{L}$ ', agitator-to-wall clearance ' $w$ ', impairs thickness 'e' and geometrical characteristics are reported in Table 1. 
Table 1. Geometrical characteristics of the agitator.

\begin{tabular}{|c|c|}
\hline D & 1 \\
\hline d & 0.96 \\
\hline da & 0.023 \\
\hline e & 0.027 \\
\hline L & 0.067 \\
\hline w & 0.02 \\
\hline
\end{tabular}

\section{Comportment law of fluid}

To study the yield stress fluids which constitute the purpose of this paper, mixing systems are dealt with a numerical simulation using Viscoplastic fluids modeled by Bingham law:

$$
\begin{aligned}
& \overline{\bar{D}}=0 \text { For }\|\tau\|<\tau_{0} \\
& \overline{\bar{\tau}}=\left(\frac{\tau_{0}}{\dot{\gamma}}+\eta_{\infty}\right) \overline{\bar{D}} \text { For }\|\overline{\bar{\tau}}\|>\tau_{0}
\end{aligned}
$$

Where $\overline{\bar{D}}$ and $\bar{\tau}$, are respectively the rate of strain tensor and the stress tensor. $\tau_{0}$ is the yield stress.

The shear rate is defined as $\dot{\gamma}=\sqrt{2 \operatorname{tr} \overline{\bar{D}}^{2}}$ where $\operatorname{tr}$ stands for the trace.

$\overline{\bar{D}}$ And $\bar{\tau}$ are defined as:

$\overline{\bar{D}}=1 / 2\left(\nabla V+\nabla V^{T}\right)$

$|\bar{\tau}|=\sqrt{\frac{1}{2} \bar{\tau}: \bar{\tau}}$

According to equations (1) and (2), the flow domain for Bingham fluid is characterized by two distinct regions. In the regions where $|\bar{\tau}| \prec \tau_{0}$ the material behaves as a solid (rigid zone), and in the regions where $|\bar{\tau}| \succ \tau_{0}$ the material flow with an apparent viscosity $\eta_{a p}$.

$$
\eta_{a p}=\eta_{\infty}+\frac{\tau_{0}}{\dot{\gamma}}
$$

The major difficulty with the constitutive equation (1) and (2) when used for numerical simulation is the discontinuity associated with infinite value of the viscosity when $|\tau|$ approaches $\tau_{0}$. This is reached closed to the yield surface delimiting plug regions. Some numerical works have been published on methods adapted to the initial set of equations [(1), (2)] completed by continuity equation and momentum equation in stress formulation. But most of the published works on this topic use modified versions which consist in replacing equations. (1) + (2) By a unique and continuous equation written with a variable viscosity $\eta_{a p}[9]$ :

$$
\tau=2 \eta_{a p} \overline{\bar{D}}
$$

\section{Model equations and computational procedure}

The equations describing fluid flow are derived from the conservation of mass and momentum which are also known as Navier-Stokes equations for fluids of constant physical properties:

$\nabla \cdot V=0$

$\rho \frac{\partial V}{\partial t}+\rho(V . \nabla)-\eta_{a p} \Delta V-2 \overline{\bar{D}} \cdot \nabla \eta_{a p}+\nabla P=0$

$\frac{\partial \theta}{\partial \tau}+U \frac{\partial \theta}{\partial X}+V \frac{\partial \theta}{\partial Y}=\frac{1}{\operatorname{Re} \cdot \operatorname{Pr}}\left(\frac{\partial^{2} \theta}{\partial X^{2}}+\frac{\partial^{2} \theta}{\partial Y^{2}}\right)$

With Prandtl number :

$\operatorname{Pr}=\frac{v}{a}$

\subsection{Boundary conditions}

In the CFD code used two reference frames can be treated. One is stationary, the impeller is in rotation and the tank is fixed. The other frame, which is rotating, the impeller is kept stationary while the outer wall of the vessel is given an angular velocity equal and opposite to the velocity of the rotating frame. Constant boundary conditions have been set respecting a fixed reference frame (RRF) approach. The boundary conditions for velocity are fixed on the gate impeller and the vessel. On the gate impeller: Moving reference frame implemented in the CFD code.

\subsection{Validation}

To verify the model of the fluid and to validate our results we compared our power number with previous works on an anchor agitator. Figure 2 gives an example of comparison between our results and a numerical work of Marouche [14]. The results show a satisfactory agreement. 


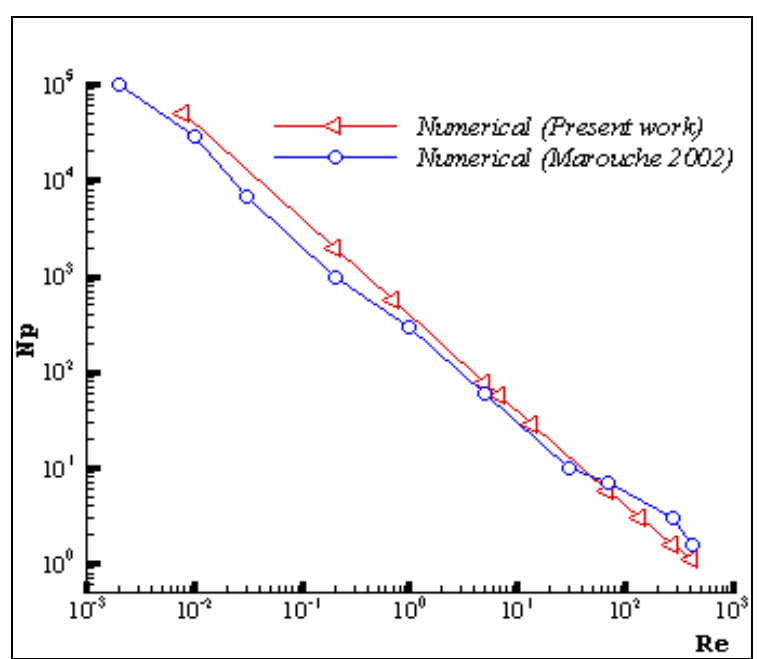

Fig. 2. Variation of power number as function of Reynolds number.

\section{Results and interpretations}

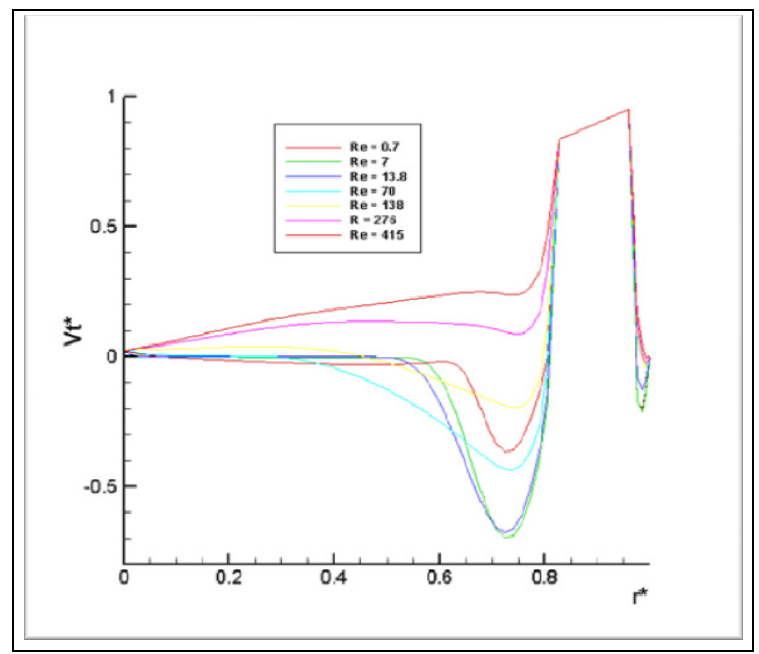

Fig. 3. Influence of inertia on the tangential velocity at the blade and its extension.

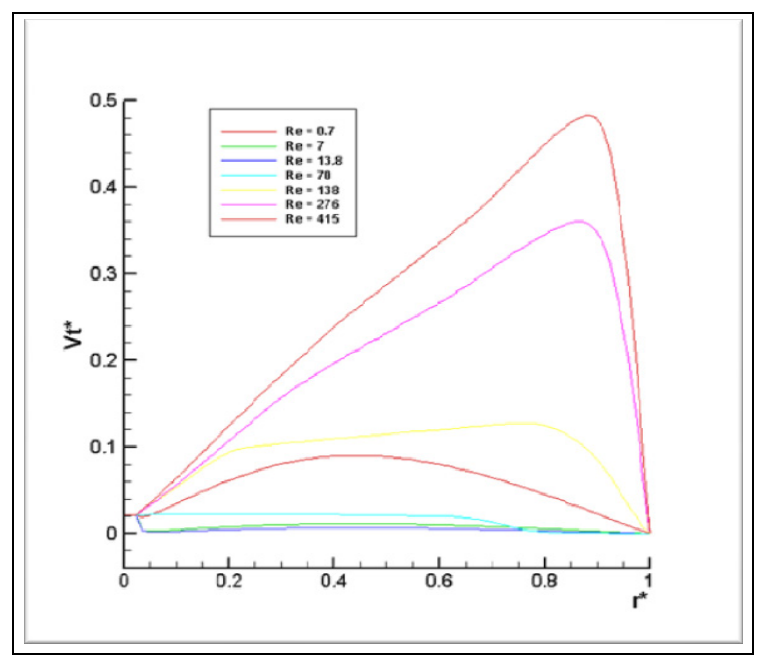

Fig. 4. Influence of inertia on the radial velocity at the blade and its extension.

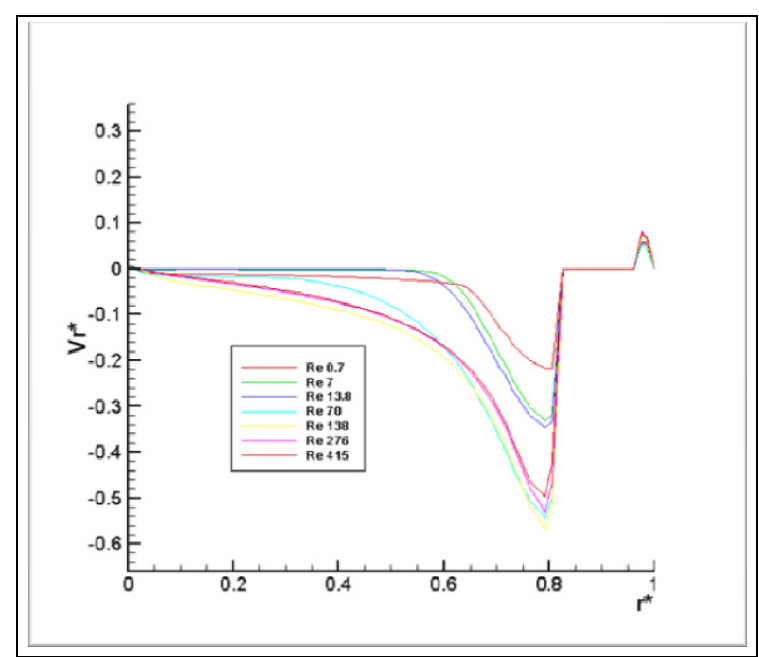

Fig. 5. Influence of inertia on the tangential velocity at the mediator.

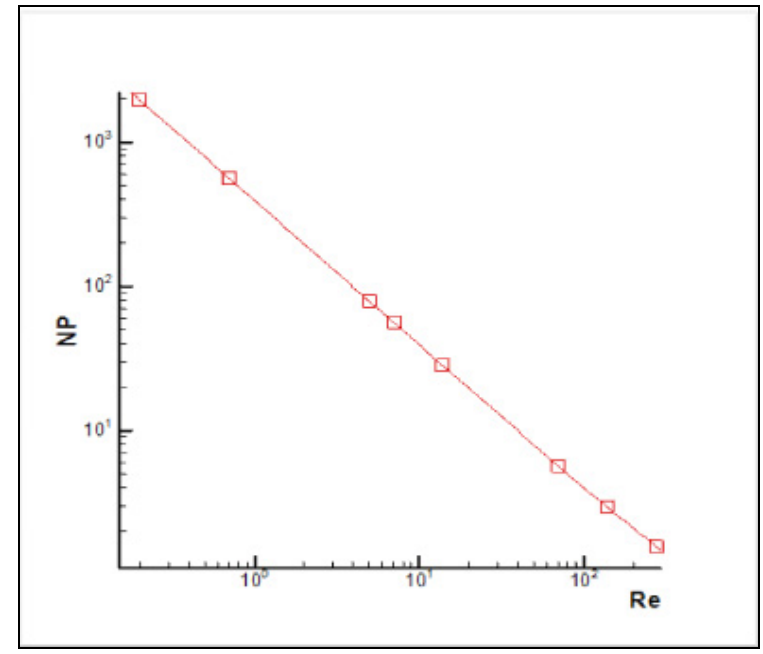

Fig. 6. Influence of the anchor on the power consumption.

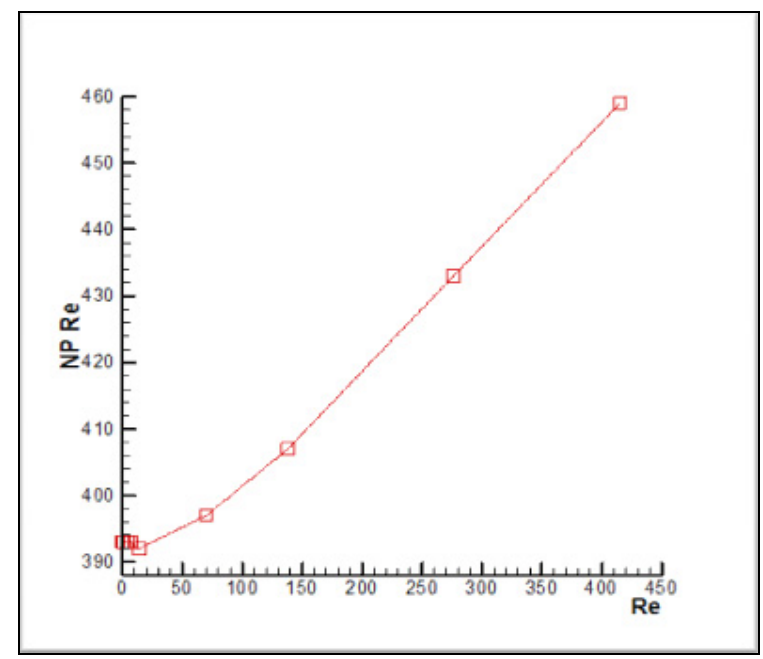

Fig. 7. Influence of the anchor on the Product $(\mathrm{Np} * \mathrm{Re})$. 


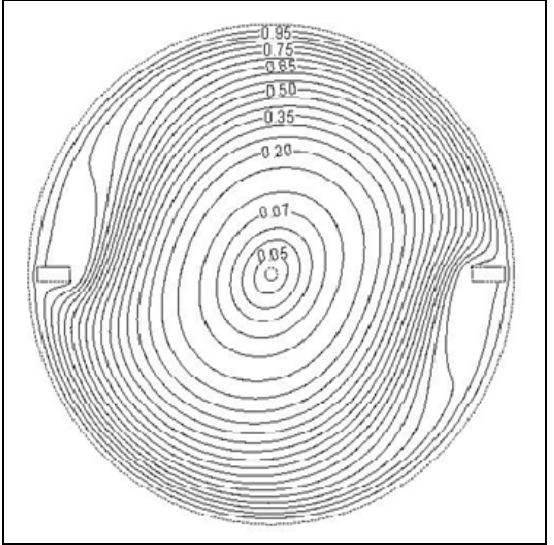

(A)

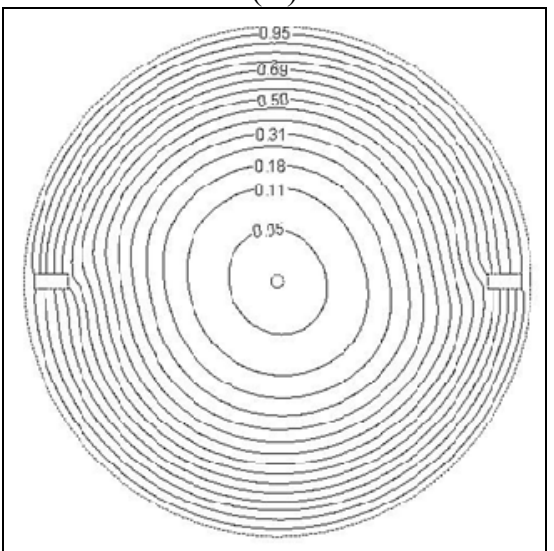

(B)

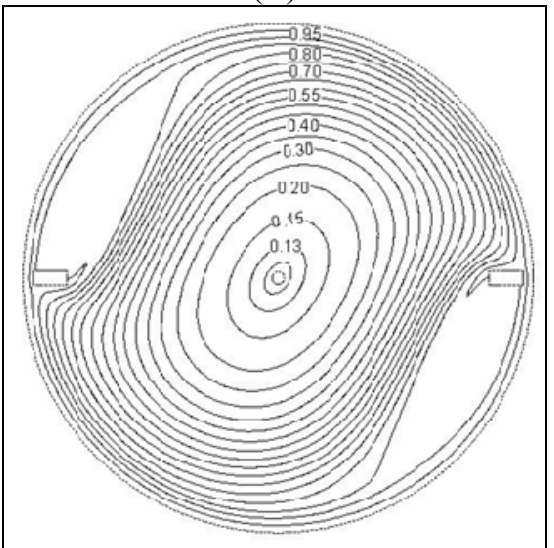

(C)

Fig. 8. Isotherms lines (a): $R e=0.7,(b): R e=13.8$, (c): $\mathrm{Re}=415$ for $\mathrm{Pr}=7$

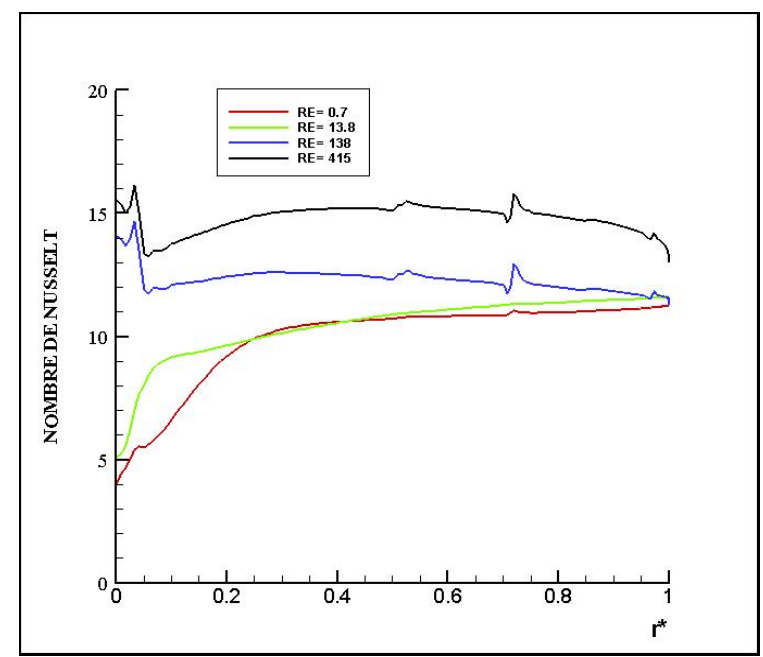

Fig. 9. Inertia effect on the Nusselt number as a function of Reynonlds number.

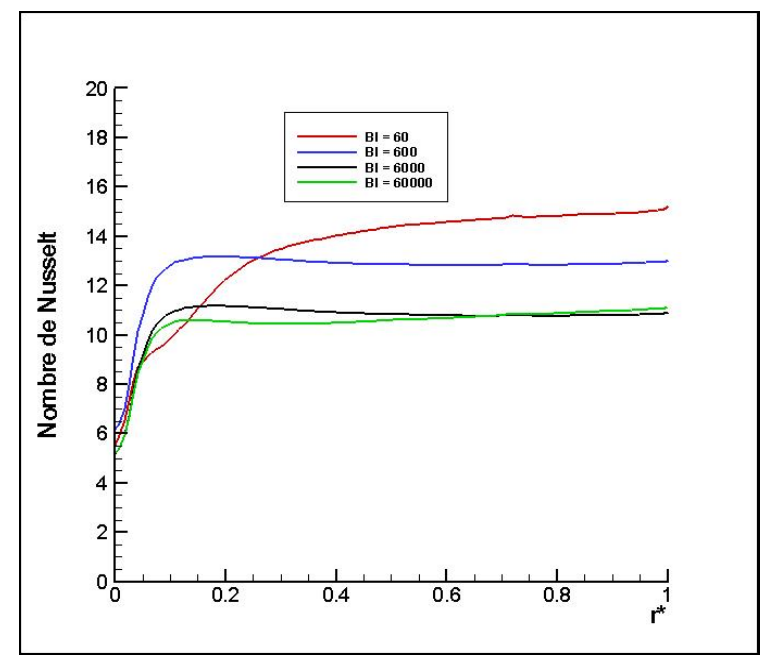

Fig. 10. Plasticity effect on the Nusselt number as a function of Bingham number.

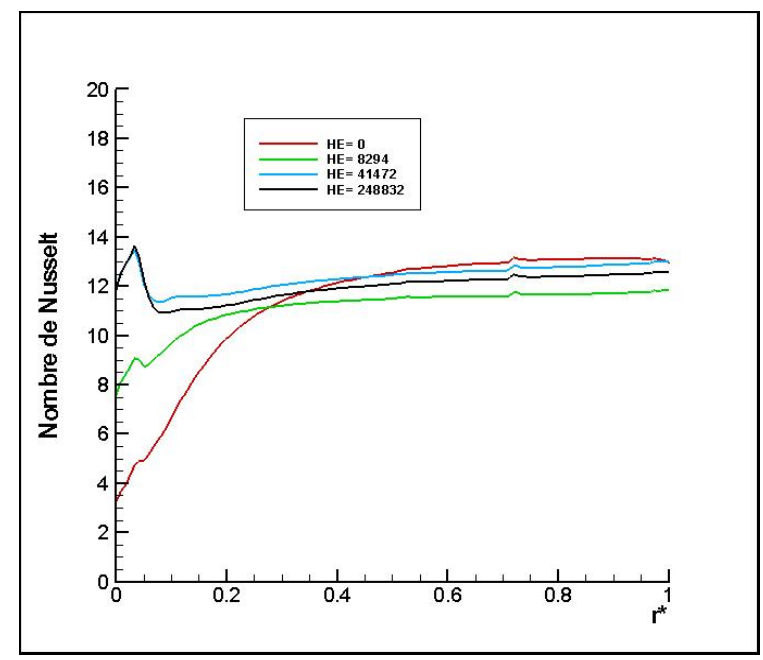

Fig. 11. Effect of Hedstrom number on the Nusselt number for different radious position.

Figures 3, 4, 5, 6 and 7 show the variation of the tangential and radial velocity of the agitator and its extension and on the mediator according to the radial 
position. We studied the influence of Reynolds number on the nature of the flow by varying this parameter from 0.7 to 415 for a Bingham fluid threshold $\tau 0=1 \mathrm{~Pa}$.

We note that the isotherms are in perfect harmony with the stream function lines, i.e. they take the same shape as the current stream lines. It is clear that these movements in the radial direction contribute to supporting heat exchange within the vessel. The trajectory of the heated fluid tends to be extended and deflected only by the action of the tangential velocities. We also find that in the immediate vicinity of the heating surface, the fluid adhering to the wall of the tank shows a large temperature gradient. On the one hand, the lowest temperatures reside within the recirculation zones. Between adjacent threads of fluid, the heat is probably conductive (Figure 8).

The thermal performance is intimately related to the hydrodynamics of the state of agitated tank. Two different processes combine to ensure homogenization: the first is a mechanical process which allows the penetration of the hot flow of the fluid inside the tank. The second is probable in the course of which the microscopic molecular diffusion due to its increase in the flow threshold is important and the differences between the lines of the current. At this stage, on the one hand, the zone or the stream lines would be very different, the temperature should be higher and on the other hand, local temperature gradients would be very large which leads to a very important exchange for the heat.

Figure (8) show the isotherms for different flow regimes. For small numbers of Reynolds they are symmetrical by contribution to the mediator, on the other hand when increasing number of Reynolds this symmetric will be disappearing.

Figure (9) illustrates the evolution of the Nusselt number as a function of the position by varying the flow regime. Note that this parameter increases by increasing the Reynolds number by 10 to 15 at most. Also notes that this parameter is higher in the wall than the center of the vessel for low numbers of Reynolds, also observes maximum local values near the blades and in the center of the tank from the recirculation zone which generated in these areas.

Figure (10) represents the evolution of the Nusselt number as a function of the position by varying the viscoplasticity, it is noted that this parameter decreases when increasing the plasticity of the fluid. The Nusselt number varies from 6 to 16 in maximum, we remark as well as from the radial position $r=0.3$ this parameter remains constant for a determined Bingham number.

The figure (11) shows the evolution of the Nusselt number as a function of the number of Hedstrom $(\mathrm{He})$, observes an increase of this parameter to the radial position $r=0.3$ with the increase of the Hedstrom number from this position, this number will be decreased slightly with the increasing of the Hedstrom number.

\section{Conclusions}

This study was motivated by the need to introduce fluid mechanics in the process of design and optimization of thermal transfer in process engineering. Our work has concentrated on viscoplastic fluids whose behaviour is still poorly controlled and which are involved in many applications.

During this process, the behaviour evolves over time: practically Newtonian at the beginning of the reaction, it becomes rheofluidifier and then viscoplastic when the concentrations increase. The non-Newtonian behaviours of the rheofluidifying type have been widely studied in the literature, and their influence studied both on the global quantities, such as the power consumed, as well as on the local variables such as the velocity field.

On the other hand, the hydrodynamics of viscoplastic fluids in complex geometries, such as agitation systems, have been little studied. However, it plays an important role in the conduct and efficiency of a process since it conditions transfers, so this work is a step in the study of these concentrated systems. We have thus studied the hydrodynamics of a model fluid for viscoplastic behaviour and heat transfer within a classical agitation system for highly viscous fluids, namely a Bingham fluid in a mechanically agitated system.

We have analyzed this problem numerically. A first work was to validate this approach with previous work. This work was coupled with an analytical study on the revolution of the power consumed and heat transfer within such a system. On this simple flow on which the inertia does not intervene, we know that the coefficient Ks of Metzner and Otto is constant when the flow is totally sheared and the fluid then has a pseudo-plastic behaviour. It tends towards a constant value for the very large numbers of Bingham.

Numerical simulations, carried out in 2D geometries, allowed to show the important unifications induced by the viscoplastic behaviour and in particular the quasi mobilization of the fluids in a large region when the threshold effects are dominant. This configuration is obviously detrimental to a heat transfer operation for the tested anchor agitator.

It has been observed in particular that the plasticity considerably reduces the axial flow. We have shown that this extension is not a simple calibration of geometry but depends on the crossed effects of the Bingham number of and Reynolds. This led us to note that for this fluid type the choice of an anchor is appropriate and consumes less power. 


\section{References}

[1] Kerim Yapici, Bulent Karasozen, Michael Schafer, Yusuf Uludag. Numerical investigation of the effect of the Rushton type turbine design factors on agitated tank flow characteristics. Chemical Engineering and Processing. (2007).

[2] F. Ein-Mozaffari, C.P.J. Bennington, G.A. Dumont, Suspension yield stress and the dynamic response of agitated pulp chests, Chem. Eng. Sci. 60 (2005).

[3] T.P. Elson, The growth of caverns formed around rotating impellers during the mixing of a yield stress fluid, Chem. Eng. 96 303-319 (1990).

[4] D. Anne-Archard, M. Marouche, H.C. Boisson. Hydrodynamics and Metzner-Otto correlation in stirred vessels for yield stress fluids. Chemical Engineering Journal, 125, 15-24 (2006).

[5] J.N. Haque, T. Mahmud, K. Roberts, Modeling turbulent flows with freesurface in unbaffled agitated vessels, Ind. Eng. Chem. Res. 45, 28812891 (2006).

[6] V.V. Ranade, S.M.S. Dommeti, Computational snapshot of flow generated by axial impellers in baffled stirred vessels, Trans. Inst. Chem. Eng. 74, 476-484 (1996).

[7] J.J. Derksen, Numerical simulation of solid suspension in a stirred tank, AIChE J. 49, 27002714 (2003).

[8] M. Marouche, Hydrodynamique d'un système d'agitation en fluide viscoplastique et en régime laminaire inertiel, Thèse de doctorat, Institut Nationale Polytechnique de Toulouse. (2002).

[9] L. Rahmanil et al. Heat transfer to Bingham fluid during laminar flow in agitated tank. International Review of Mechanical Engineering (I.RE.M.E.), Vol. 09, (2013).

[10] O'Donovan EJ, Tanner RI, Numerical analysis of the Bingham squeeze film problem, journal of NonNew. Flu. Mec. 15 ,75-83, (1984).

[11] PATANKAR S. V. Numerical heat transfer and fluid flow. Mc-Graw Hill. (1980).

[12] G.C. Vradis, M.V. Otugen, The axisymmetric sudden expansion flow of a non-Newtonian viscoplastic fluid, J. Fluid Eng. 119, 193-200 (1997).

[13] M. Marouche, D. Anne-Archard, H.C. Boisson, A numerical model of yield stress fluid dynamics in a mixing vessel, Appl. Rheol. 12, 182-191 (2002).

[14] M. Marouche, Hydrodynamique d'un système d'agitation en fluide viscoplastique et en régime laminaire inertiel, Thèse de Doctorat de l'INP Toulouse, France, (2002).

[15] A.B. Metzner, R.E. Otto, Agitation of nonNewtonian fluids, AIChE J. 3, 3-10 (1957). 